\title{
Comparison of clinico-pathological characteristics and survival of recurrent ovarian cancer patients on seven different chemo-protocols
}

\author{
SAIMA SATTAR ${ }^{1}$ \\ MOBASHER AHMAD ${ }^{1}$ \\ HAMID SAEED $^{1^{*}}$ \\ ZIKRIA SALEEM ${ }^{1}$ \\ ZEESHAN DANISH ${ }^{1}$ \\ MUHAMMAD AKHLAQ 2 \\ ${ }^{1}$ Section of Clinical Pharmacy \\ University College of Pharmacy \\ University of the Punjab \\ Allama Iqbal Campus \\ 54000, Lahore, Pakistan \\ ${ }^{2}$ Department of Pharmaceutics \\ Faculty of Pharmacy \\ Gomal University \\ Dera Ismail Khan \\ Khyber Pakhtunkhwa, Pakistan
}

Accepted July 16, 2018

Published online August 10, 2018

\begin{abstract}
Despite growing prevalence of ovarian cancer (OC) in Pakistan, no literature evidence exists regarding its clinic-pathological characteristics, survival and compliance of patients with recurrent ovarian cancer on various chemo-protocols. An observational study was conducted by enrolling 251 recurrent OC patients on 7 different chemo-protocols, from a specialized cancer care hospital, Lahore, Pakistan, using convenient judgmental sampling. The study was conducted for a period of 6 months. Most of the patients were between 18 and 70 years of age, with IIIC FIGO stage and papillary serous histological grade. As per RECIST, improved partial response (PR) (63.3\%) and complete response (CR) $(52.1 \%$ ) was observed in the CP (carboplatin + paclitaxel) arm, substantiated by improved median progression free survival (PFS) and overall survival (OS) in $\mathrm{CP}$ and $\mathrm{CD}$ (carboplatin + docetaxel) arms, respectively, yet with no significant differences in survival curves, PFS ( $p=$ $0.12)$ and OS $(p=0.22)$. Interestingly, the highest and the lowest patient non-compliance were observed in CG (carboplatin + gemcitabine) $(81.6 \%$ ) and paclitaxel (4.5\%) arms, resp. As per the hazard model for survival, topotecan showed significant association with the therapy related events/deaths compared to other protocols. These data suggest that $\mathrm{CP}$ regimen exhibited improved clinical efficacy and decreased toxicity related non-compliance in recurrent ovarian cancer patients of Lahore.
\end{abstract}

Keywords: ovarian cancer, carboplatin plus paclitaxel, topotecan, bevacizumab, docetaxel

In women, ovarian cancer (OC) is the fourth most common cause of death and sixth most common type of cancer - making it a leading cause of mortality from all gynecological malignancies $(1,2)$. In the majority of cases, ovarian cancer, rare before the age of

\footnotetext{
*Correspondence; e-mail: hamid.pharmacy@pu.edu.pk
} 
40 , is predominantly diagnosed in elderly postmenopausal women above 50 years of age (3). In routine clinical practice, OC diagnosis is made incidentally during the work-up of another clinical condition, while symptoms remain poorly defined with no definite indices to screen the asymptomatic women; however, if confined to ovaries, it is highly curable with improved five-year survival (4). Most patients are referred to the clinics with an advanced stage of disease - FIGO (International Federation of Gynecological Oncology) stage III and IV, requiring aggressive surgery and chemotherapy (5).

Over the last several years, carboplatin plus platinum based regimen has been the standard first line chemotherapy in ovarian cancer. However, despite 60-80\% response rate with carboplatin and paclitaxel, the majority of patients subsequently relapse and need further clinical management $(3,6)$. In this context, in previous years, numerous trials (GOG182, ICON5) have been conducted to add a third drug to the chemotherapeutic regimen in an attempt to increase overall survival (OS) (7). However, despite an increase in the number of therapies, the clinical judgment about treatment choices in recurrent cases has become even more complex. In this regard, the results of clinical trials and observational studies are becoming increasingly important in clinical decision making on managing the advanced stage disease.

Clinical management of ovarian cancer, just like other malignancies, has evolved from a single agent to a combination therapy. A number of studies have been conducted to compare several available chemotherapeutic options in an attempt to find an appropriate chemotherapeutic option for patients with recurrent disease $(8,9)$. Among the South Asian countries, ovarian cancer is reported to be higher in Pakistan $(10,11)$ and is the third most common malignancy in Pakistani women (12). Very few studies, prospective or observational, evaluated and compared clinical responses of seven available chemotherapeutic regimens used in the treatment of recurrent ovarian cancer patients. The present study is the first report that has compared the clinic-pathological characteristics, clinical responses, therapy compliance and toxicity of several chemo-therapeutic agents in recurrent ovarian cancer patients of Lahore, Pakistan.

\section{EXPERIMENTAL}

\section{Ethical approval}

The study was duly approved by the Ethical Committee of Clinical Research, University College of Pharmacy, University of the Punjab, Lahore, Pakistan, and the Hospital Committee of Ethics on Human Research of the Shaukat Khanum Memorial Cancer Hospital \& Research Center, Lahore, Pakistan.

\section{Study design and population}

The observational study was designed by enrolling a total of 251 recurrent ovarian cancer patients from Lahore, Pakistan. The study period was six months, from August 2015 to January 2016, in a specialized cancer care hospital. Convenient judgmental sampling was used to enroll patients to avoid missing data. Data was retrieved from the hospital information system (HIS). Study sample size was 181, calculation based on ovarian cancer prevalence in Pakistan, i.e., 13.6 \% (13) using $95 \%$ confidence interval and $5 \%$ margin of 
error. However, more patients were enrolled for improved reliability and statistical power. Enrolled OC patients were segregated into 7 chemo-arms, i.e., paclitaxel (PTX), topotecan (TOPO), carboplatin + liposomal doxorubicin (CLD), carboplatin + gemcitabine (CG), carboplatin + paclitaxel $(\mathrm{CP})$, carboplatin + docetaxel (CD), carboplatin + gemcitabine + bevacizumab (CGB).

All ovarian cancer patients with conformed diagnosis as per the International Federation of Gynecology and Obstetrics (FIGO) staging system (Table SI) and conforming inclusion/exclusion criteria were included in the study.

Inclusion criteria. - All recurrent OC patients, above 18 years of age, irrespective of religion, race and ethnicity, disease stage, with normal hepatic and renal functions, who had given consent for their participation in research studies were included in the study.

Exclusion criteria. - Women below 18 years of age having compromised renal and hepatic functions, incomplete chemotherapy cycles and who failed to provide informed consent were excluded from the study.

\section{Data collection}

A data collection form was designed to fulfill all the necessary objectives of the study. Clinical data included patients' basic demographics, histology subtype and FIGO staging, details of chemotherapeutic protocols, patient non-compliance due to interruptions or toxicity, therapy responses and relevant laboratory findings. All treatment plans, dose, frequency and cycles, are summarized in supplementary materials (Table SI).

\section{Outcome measures}

Primary end points of the study were progression-free survival (PFS) and rate of overall survival (OS) (14). Clinical response was evaluated both by the tumor marker CA-125 levels and radiological examination using new response evaluation criteria in solid tumor (RECIST) guidelines (supplementary material, Table SII) (15). Laboratory values were assessed at a baseline and after every chemotherapy cycle. Adverse events were graded according to the NCI common terminology criteria for adverse events (NCI-CTCAE), version 3.0 (16). Patient compliance was assessed by treatment interruptions and delays due to disease progression, death and toxicity.

\section{Data analysis}

All statistical analyses were performed using Graphpad (Prism5) and SPSS (IBM, version 21). PFS and OS curves were estimated by the Kaplan-Meier method and compared using a log-rank test (17). Frequency of untoward events/deaths during chemotherapy was determined by the Cox proportional hazard model for PFS and OS (18). Laboratory mean values in all the chemotherapy protocols were compared by the analysis of variance (ANOVA). $p$-value of 0.05 or less was considered statistically significant.

\section{RESULTS AND DISCUSSION}

Clinical characteristics of OC patients are summarized in Table I. The median age ranged from $48.5-55$ years in all therapeutic arms (Table I). Surprisingly, compared to our findings 
of the early age (median 48 years) reporting of recurrent OC in women of Lahore, Pakistan, the reporting age of women was usually above 55 years in Europe and US $(18,19)$. It is also plausible that young women of Lahore, Pakistan, are exposed to specific or increased risks earlier in their life for an unascertained period of time of known or unknown origin. Irrespective of chemo-protocols, stage IIIC was the most frequently reported FIGO stage with the following frequency distribution within the respective arms, i.e., paclitaxel (PTX): 64.2 $\%$, topotecan (TOPO): $42.4 \%$, carboplatin + liposomal doxorubicin (CLD): $42.8 \%$, carboplatin + gemcitabine (CG): $66.6 \%$, carboplatin + paclitaxel (CP): $52.2 \%$, carboplatin + docetaxel (CD): $53.3 \%$ and carboplatin + gemcitabine + bivacizumab (CGB): $60.3 \%$, followed by stage IV (Table I). Likewise, papillary serous was the most common histological stage (PTX: 27.2 \%, TOPO: $39.3 \%$, CLD: $24.0 \%$, CG: $16.6 \%$, CP: $16.7 \%$, CD: $13.3 \%$ and CGB: $30.0 \%$ ), followed by poorly differentiated histology (Table I). The above mentioned findings corroborate many other reports $(20,21)$, suggesting that irrespective of ethnicity and geographical region, ovarian cancer clinical presentations exhibit minimal differences.

Lately, with the introduction of paclitaxel (21), carboplatin plus paclitaxel has become the standard combination therapy in advanced and recurrent ovarian cancer (platinum sensitive) $(22,23)$. In a systematic review, Fung-Kee-Fung et al. (24) found that in five out of thirteen trials almost all patients were platinum sensitive and re-treatment with platinum based combination significantly improved PFS and OS. Combination of a platinum based drug with either paclitaxel or gemcitabine has been shown to improve the response rate in

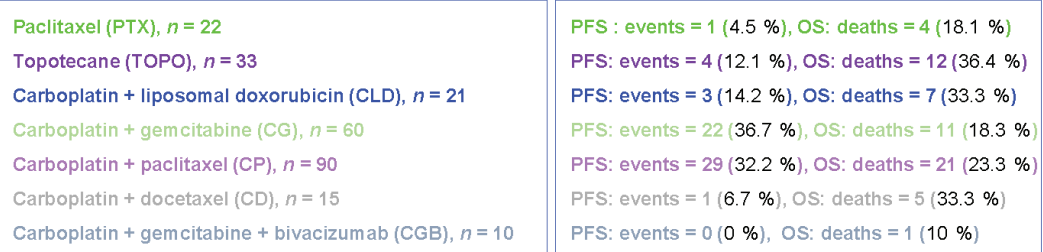

a)

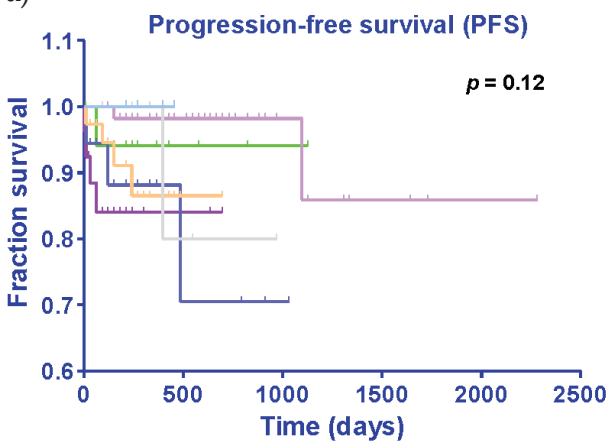

b)

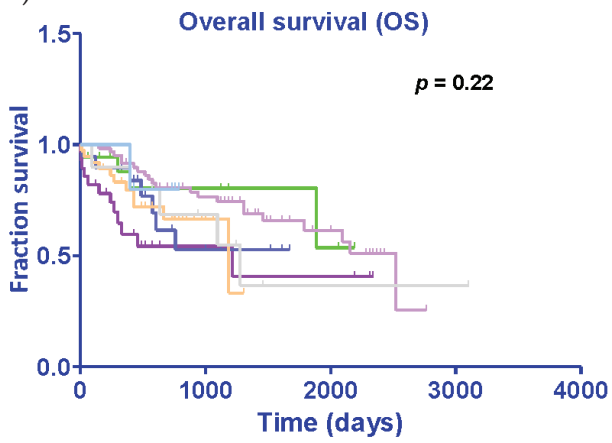

Fig. 1. Kaplan-Meier survival curves for all seven protocols: a) progression-free survival (PFS) curves for all seven chemo-protocols compared by the log-rank test. Death reported during the period of therapy was considered an event, b) overall survival curves for all seven protocols compared by the log-rank test. 
Characteristic

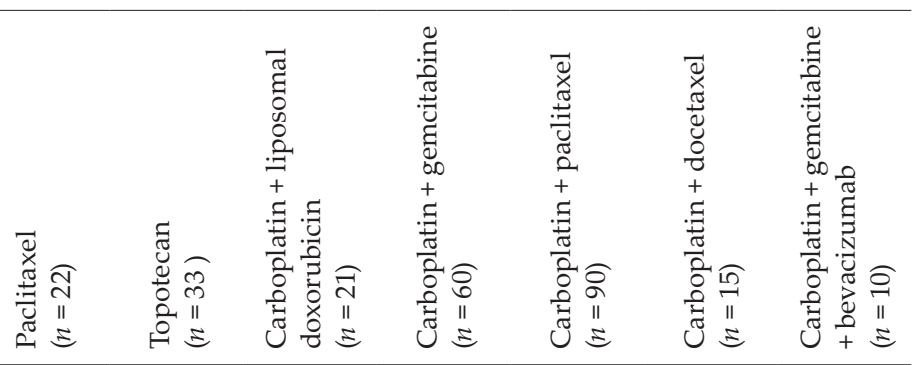

\begin{tabular}{|c|c|c|c|c|c|c|c|}
\hline \multicolumn{8}{|c|}{ Patient clinico-pathological characteristics $(n, \%)$} \\
\hline $\begin{array}{l}\text { Median age } \\
\text { (range) }\end{array}$ & $\begin{array}{c}51 \\
(20-65)\end{array}$ & $\begin{array}{c}55 \\
(18-70)\end{array}$ & $\begin{array}{c}51 \\
(32-66)\end{array}$ & $\begin{array}{c}49.5 \\
(20-61)\end{array}$ & $\begin{array}{c}50 \\
(20-68)\end{array}$ & $\begin{array}{c}49 \\
(29-60)\end{array}$ & $\begin{array}{c}48.5 \\
(33-64)\end{array}$ \\
\hline \multicolumn{8}{|l|}{ Marital status } \\
\hline Married & 21 (95) & 32 (97) & 20 (95) & 57 (95) & $78(87)$ & 14 (93) & $10(100)$ \\
\hline Unmarried & $1(5)$ & $1(3)$ & $1(5)$ & $3(5)$ & $12(13)$ & $1(7)$ & $0(0)$ \\
\hline \multicolumn{8}{|l|}{ FIGO stage } \\
\hline IA & 0 & 0 & $1(5)$ & $2(3)$ & $5(5)$ & 0 & 0 \\
\hline IB & 0 & 0 & $1(5)$ & $2(3)$ & 0 & $2(13)$ & 0 \\
\hline IC & $1(5)$ & $1(3)$ & 0 & $2(3)$ & $7(8)$ & 0 & 0 \\
\hline IIA & 0 & 0 & $1(5)$ & $1(2)$ & $6(5)$ & $1(7)$ & $1(10)$ \\
\hline IIB & 0 & 0 & $1(5)$ & $2(3)$ & $7(8)$ & 0 & 0 \\
\hline IIIA & 0 & $1(3)$ & $2(10)$ & 0 & $1(1)$ & $1(7)$ & 0 \\
\hline IIIB & $1(5)$ & 0 & $1(5)$ & $1(2)$ & $2(2)$ & $1(7)$ & 0 \\
\hline IIIC & $14(64)$ & $14(42)$ & $9(42)$ & $40(67)$ & $47(52)$ & $8(53)$ & $6(60)$ \\
\hline IV & $6(27)$ & $13(40)$ & $5(23)$ & $10(17)$ & $15(17)$ & $2(13)$ & $3(30)$ \\
\hline Missing & 0 & $4(12)$ & 0 & 0 & 0 & 0 & 0 \\
\hline \multicolumn{8}{|l|}{ Histo-pathology } \\
\hline Endometrioid & $2(9)$ & $1(3)$ & $3(14)$ & $11(18)$ & $17(18)$ & $2(13)$ & 0 \\
\hline Poorly differentiated & $7(32)$ & $7(21)$ & $5(24)$ & $7(12)$ & $19(21)$ & $2(13)$ & $2(20)$ \\
\hline Serous & $1(4)$ & $2(6)$ & $2(9)$ & 7 (12) & $11(12)$ & $2(13)$ & $3(30)$ \\
\hline Papillary serous & $6(27)$ & $13(39)$ & $7(33)$ & $15(25)$ & $19(21)$ & $5(33)$ & $2(20)$ \\
\hline High grade serous & $3(14)$ & $1(3)$ & $1(5)$ & $11(18)$ & $5(6)$ & $3(20)$ & $3(30)$ \\
\hline Mucinous & 0 & $4(12)$ & 0 & 0 & $5(6)$ & $1(7)$ & 0 \\
\hline Clear cell & $2(9)$ & $3(9)$ & $1(5)$ & $4(7)$ & $6(7)$ & 0 & 0 \\
\hline Missing & $1(4)$ & $2(6)$ & $2(9)$ & $5(8)$ & $8(9)$ & 0 & 0 \\
\hline
\end{tabular}

FIGO - International Federation of Gynecology and Obstetrics 


\begin{tabular}{|c|c|c|c|c|c|c|c|}
\hline 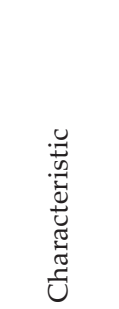 & 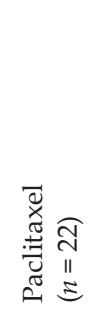 & 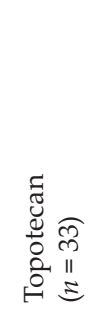 & 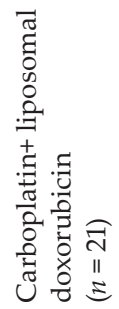 & 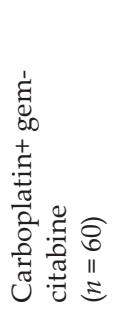 & 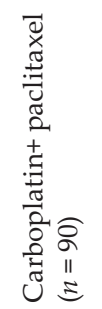 & 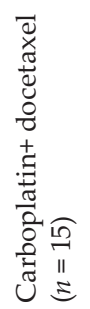 & 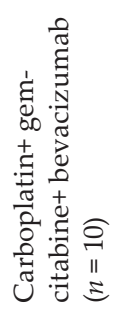 \\
\hline \multicolumn{8}{|c|}{ Response to chemotherapy: $n(\%)$} \\
\hline \multicolumn{8}{|c|}{ After 3 cycles } \\
\hline PR & $11(50)$ & $10(30)$ & $11(52)$ & $31(52)$ & $57(63)$ & $5(33)$ & $8(80)$ \\
\hline $\mathrm{CR}$ & $1(4)$ & - & $1(5)$ & $2(3)$ & $16(18)$ & - & - \\
\hline SD & $5(23)$ & $6(18)$ & $6(29)$ & $11(18)$ & $11(12)$ & $6(41)$ & $2(20)$ \\
\hline PD & $4(18)$ & $12(36)$ & $3(14)$ & $7(12)$ & $2(3)$ & $2(13)$ & - \\
\hline Missing & $1(4)$ & $5(15)$ & - & $9(15)$ & $4(4)$ & $2(13)$ & - \\
\hline \multicolumn{8}{|c|}{ After 6 cycles } \\
\hline PR & $8(36)$ & $6(18)$ & $6(28)$ & $19(31)$ & $19(21)$ & $4(27)$ & $3(30)$ \\
\hline CR & $3(13)$ & $1(3)$ & $3(14)$ & $4(7)$ & $47(52)$ & $3(20)$ & $1(10)$ \\
\hline SD & $2(9)$ & $4(12)$ & 4 (19) & $12(20)$ & $10(11)$ & $3(20)$ & $5(50)$ \\
\hline PD & $6(27)$ & $19(57)$ & $5(24)$ & $19(32)$ & $11(12)$ & $3(20)$ & $1(10)$ \\
\hline Missing & $3(14)$ & $3(9)$ & $3(14)$ & $6(10)$ & $3(3)$ & $2(13)$ & - \\
\hline
\end{tabular}

$\mathrm{CR}$ - complete response, $\mathrm{PD}$ - progressive disease, $\mathrm{PR}$ - partial response, RECIST - response evaluation criteria in solid tumors, SD - stable disease

patients with recurrent ovarian cancer $(9,22)$. In our study, clinical response to chemotherapy protocols was assessed according to RECIST (supplementary material, Table SII). After the $3^{\text {rd }}$ cycle, partial response (PR) was observed in $62 \%$ patients belonging to $\mathrm{CP}$ and CGB arms while it was $\sim 50 \%$ in paclitaxel, CLD and CG arms (Table II). After the $3^{\text {rd }}$ cycle, stable disease (SD) frequency was observed in $40 \%$ patients in CD, $28.5 \%$ in CLD and $22.7 \%$ in the paclitaxel arm. After the $6^{\text {th }}$ cycle, CR frequency improved slightly in all the treatment arms - more markedly in the CP (52\%) arm, but with reduced PR (Table II). Progressive disease (PD) frequency increased markedly in paclitaxel (27\%), topotecan (57 $\%)$, CLD (23.8 \%) and CG (31.7 \%) arms (Table II). More recently, the CD combination has been employed in platinum sensitive recurrent ovarian cancer with the overall response rate of $70 \%$, CR of $28 \%$ and median PFS of 12.4 months (25) - complemented by another report with $\mathrm{CR}$ of $38 \%$ employing $\mathrm{CD}$ regimen (26), but with hematological toxicities, i.e., neutropenia. In our data, after the $6^{\text {th }}$ cycle, compared to the $C P$ arm $(C R, 52 \%)$, the CD arm demonstrated CR in $20 \%$ of patients, but also with higher hematological toxicities. Similarly to our findings with $52 \%$ complete response (CR) in the CP arm, Gibson et al. (27) found CR of $40-60 \%$ in patients on CP regimen. 
As shown in Fig. 1, no significant differences were observed between patients' survival PFS (log-rank test, $p=0.12$ ) and OS (log-rank test, $p=0.22$ ) on various chemo-protocols (Fig. 1). The highest median progression-free survival (PFS) was observed in the CP arm (15 months) followed by CGB (13 months), CD (11.5 months), CLD (9 months), CG (8 months), PTX (7.5 months) and TOPO (5 months) arms. Nevertheless, the median overall survival was highest in the CD arm (21 months) and lowest in the CG arm (8 months). Similarly to our findings, the ICON4/OVAR2.2 trial demonstrated that addition of paclitaxel to carboplatin extended the PFS and OS in ovarian cancer patients (28). However, when we compared PFS and OS in CP and CG arms, no significant differences were observed; yet, the maximum non-compliance frequency was observed in the CG (81.6 \%) arm, presumably due to grade 1-3 hematological toxicities. Likewise, the AGO-OVAR-led intergroup trial in recurrent ovarian cancer patients compared CG with CP regimens, demonstrating similar PFS with no improvement in OS, but with treatment delays that were attributed to myelo-suppression (29). Other studies reported that CLD was as efficacious as CP (20, 30); however, in the MITO-2 trial, despite no improvements in PFS and OS, pegylated liposomal doxorubicin (PLD) plus carboplatin was suggested as an alternative first line chemo regimen comparable to the CP regimen (19) taking into account the toxicity profiles and observed confidence intervals.

Table III. Patient compliance for various chemotherapy protocols

\begin{tabular}{|c|c|c|c|c|c|c|c|}
\hline \multicolumn{8}{|c|}{ Chemotherapy Compliance during Treatment } \\
\hline 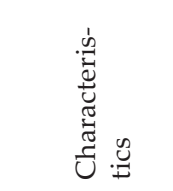 & 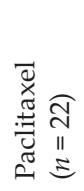 & 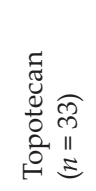 & 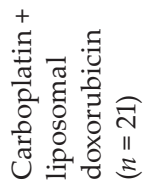 & 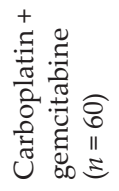 & 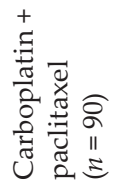 & 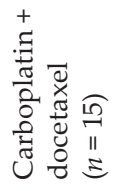 & 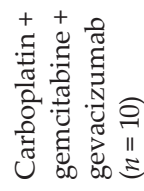 \\
\hline \multicolumn{8}{|c|}{ Cause of treatment interruption $(n, \%)$} \\
\hline $\begin{array}{l}\text { Missing } \\
\text { information }\end{array}$ & - & - & - & - & $3(3)$ & $1(7)$ & $1(10)$ \\
\hline $\begin{array}{l}\text { Progression/ } \\
\text { death }\end{array}$ & $3(14)$ & $14(42)$ & $3(14)$ & $10(16)$ & $5(6)$ & $3(20)$ & - \\
\hline Toxicity & $1(4)$ & $2(6)$ & $4(19)$ & $37(62)$ & $29(32)$ & $7(46)$ & $6(60)$ \\
\hline Others & 0 & $6(18)$ & $7(20)$ & $12(20)$ & - & $2(13)$ & $1(10)$ \\
\hline \multicolumn{8}{|c|}{ Toxicity delays } \\
\hline $2^{\text {nd }}$ cycle & 0 & 0 & 1 & 20 & 4 & 2 & 2 \\
\hline $3^{\text {rd }}$ cycle & 0 & 1 & 2 & 5 & 6 & 2 & 3 \\
\hline $4^{\text {th }}$ cycle & 0 & 1 & 0 & 3 & 5 & 1 & 0 \\
\hline $5^{\text {th }}$ cycle & 1 & 0 & 1 & 6 & 9 & 0 & 1 \\
\hline $6^{\text {th }}$ cycle & 0 & 0 & 0 & 3 & 5 & 0 & 0 \\
\hline \multirow{2}{*}{$\begin{array}{l}\text { Non-compli- } \\
\text { ance (\%) }\end{array}$} & $1 / 22$ & $8 / 33$ & $11 / 21$ & $49 / 60$ & 29/90 & 9/15 & $7 / 10$ \\
\hline & (4) & (24) & (52) & $(81)$ & (32) & (60) & (70) \\
\hline
\end{tabular}

Percent of non-compliance was estimated based on toxicity delays in each cycle and any other reason of interruption (not reporting to clinic) in treatment without taking into account reported death. 
As for therapy compliance, the proportion of treatment delays due to disease progression/deaths was highest in topotecan (42.4\%), while due to toxicity, it was highest in the carboplatin + gemcitabine arm (61.6\%) (Table III). In the combination regimen, the highest treatment non-compliance was observed in the carboplatin + gemcitabine arm $(81.6 \%)$, while the $\mathrm{CP}$ arm exhibited the lowest non-compliance frequency (32.2\%) (Table III). Thus, we observed better compliance in mono therapy (paclitaxel: $4.54 \%$, topotecan: $24.2 \%$ ) compared to combination therapy; nevertheless, no single therapy (topotecan or paclitaxel) exhibited clinical edge over the combination therapy, but there were subtle differences in treatment-related adverse events. However, toxicity with topotecan is subject to several factors, such as dose schedule adjustments based on the patient's prior treatment, radiation therapy, extent of cumulative myelo-suppression with prior agents (31). More recently, bevacizumab has been used in combination with other chemotherapeutic agents in recurrent ovarian cancer (32) with improved tolerability and encouraging efficacy. In the OCEANS trial, PFS was improved in the CGB arm compared to CG arm with high grade hypertension in the bevacizumab arm, duly supported by our findings of grade 2 hypertension in $30 \%$ subjects belonging to the CGB arm (17).

In the multivariate analysis for PFS and OS, only topotecan was significantly associated with higher rate of events [for PFS: Cox proportional hazard ratio (HR) was 20.22, confidence interval (CI) 2.16-188.94, $p=0.008$, for OS: HR was 3.83; CI 1.804-8.119, $p=0.0005$ ) (Table IV)]. Notably, significant differences were observed in laboratory values among

Table IV. Cox proportional hazard model for PFS and OS

\begin{tabular}{|c|c|c|c|c|}
\hline Factor & $\mathrm{N}$ & Hazard ratio & $95 \%$ CI & $p$-value \\
\hline Protocols & \multicolumn{4}{|c|}{$\begin{array}{l}\text { Cox proportional hazard model for progression free } \\
\text { survival (PFS) }\end{array}$} \\
\hline Carboplatin + paclitaxel & 90 & & & Reference \\
\hline Paclitaxel & 22 & - & - & - \\
\hline Topotecan & 33 & 20.2 & $(2.16-188.94)$ & $0.008^{*}$ \\
\hline Carboplatin + liposomal doxorubicin & 21 & 5.8 & $(0.36-93.99)$ & 0.21 \\
\hline Carboplatin + gemcitabine & 60 & 8.1 & $(0.72-89.54)$ & 0.08 \\
\hline Carboplatin + docetaxel & 15 & 6.3 & $(0.64-62.07)$ & 0.11 \\
\hline Carboplatin + gemcitabine + bevacizumab & 10 & - & - & - \\
\hline Protocols & \multicolumn{4}{|c|}{ Cox proportional hazard model for overall survival (OS) } \\
\hline Carboplatin + paclitaxel & 90 & & & Reference \\
\hline Paclitaxel & 22 & 0.9 & $(0.33-2.86)$ & 0.96 \\
\hline Topotecan & 33 & 3.8 & $(1.80-8.11)$ & $0.0005^{* *}$ \\
\hline Carboplatin + liposomal doxorubicin & 21 & 1.3 & $(0.44-3.88)$ & 0.61 \\
\hline Carboplatin + gemcitabine & 60 & 1.3 & $(0.54-3.40)$ & 0.51 \\
\hline Carboplatin + docetaxel & 15 & 1.3 & $(0.60-3.25)$ & 0.43 \\
\hline Carboplatin + gemcitabine + bevacizumab & 10 & 0.9 & $(0.12-7.22)$ & 0.96 \\
\hline
\end{tabular}

${ }^{*} p \leq 0.05,{ }^{* *} p \leq 0.001$

$(-)$ - no output recorded/obtained in the model. 
various chemo-protocols, such as platelets, neutrophils, hemoglobin, albumin, AST and ALT $(p<0.025)$ (supplementary material, Table SIII). Very few grade-4 toxicities, hematological and hepatic, were observed in paclitaxel, CG and CP treatment arms (supplementary material, Table SIV).

\section{CONCLUSIONS}

In conclusion, our data suggest that $\mathrm{CP}$ chemo-protocol demonstrated better median PFS and OS in recurrent ovarian cancer patients, yet statistically non-significant, along with maximum partial and complete responses after the $3^{\text {rd }}$ and $6^{\text {th }}$ cycles, resp. In the combination regimen, $\mathrm{CP}$ was associated with the lowest non-compliance, while the highest non-compliance was observed in the CG arm. However, the CP arm was associated with considerable hematological and abdominal adverse effects in addition to peripheral neuropathy and fatigue. Topotecan was associated with significantly higher propensity of having therapy related events/deaths.

Acknowledgements. - The authors are grateful to the hospital administration and patients for their kind cooperation and informed consent, helpful in completing the study. Supplementary materials are available upon request.

\section{REFERENCES}

1. B. M. Reid, J. B. Permuth and T. A. Sellers, Epidemiology of ovarian cancer: a review, Cancer Biol. Med. 14 (2017) 9-32; https://doi.org/10.20892/j.issn.2095-3941.2016.0084

2. J. Permuth-Wey and T. A. Sellers, 20. Epidemiology of Ovarian Cancer, in Cancer Epidemiology: Modifiable Factors (Ed. M. Verma)/Series: Methods of Molecular Biology 472 (2009) 413-437, Springer, New York 2009; https://doi.org/10.1007/978-1-60327-492-0_20

3. J. Ledermann, F. Raja, C. Fotopoulou, A. Gonzalez-Martin, N. Colombo and C. Sessa (ESMO guidelines working group), Newly diagnosed and relapsed epithelial ovarian carcinoma: ESMO Clinical Practice Guidelines for Diagnosis, Treatment and Follow-up, Ann. Oncol. 24 (Suppl. 6) (2013) vi24-vi32. https://doi.org/10.1093/annonc/mdt333

4. F. Raja, N. Chopra and J. Ledermann, Optimal first-line treatment in ovarian cancer, Ann. Oncol. 23 (Suppl. 10) (2012) x118-x127; https://doi.org/10.1093/annonc/mds315

5. M. Cristea, E. Han, L. Salmon and R. J. Morgan, Review: Practical considerations in ovarian cancer chemotherapy, Ther. Adv. Med. Oncol. 2 (2010) 175-187; https://doi.org/10.1177/1758834010361333

6. M. Kyrgiou, G. Salanti, N. Pavlidis, E. Paraskevaidi and J. P. Ioannidis, Survival benefits with diverse chemotherapy regimens for ovarian cancer: meta-analysis of multiple treatments, J. Natl. Cancer Inst. 98 (2006) 1655-1663; https://doi.org/10.1093/jnci/djj443

7. M. A. Bookman (Ed.), GOG0182-ICON5: 5-arm phase III randomized trial of paclitaxel (P) and carboplatin (C) vs combinations with gemcitabine (G), PEG-lipososomal doxorubicin (D), or topotecan (T) in patients (pts) with advanced-stage epithelial ovarian (EOC) or primary peritoneal (PPC) carcinoma, J. Clin. Oncol. 24 (Suppl.) (2006) 5002-5002 (ASCO Annual Meeting Proceedings 2006); https://doi.org/10.1200/jco.2006.24.18_suppl.5002

8. J. A. Ledermann and R. S. Kristeleit, Optimal treatment for relapsing ovarian cancer, Ann. Oncol. 21 (Suppl. 7) (2010) vii218-222; https://doi.org/10.1093/annonc/mdq377

9. D. S. Dizon, M. L. Hensley, E. A. Poynor, P. Sabbatini, C. Aghajanian, A. E. Hummer. D. R. Venkatraman and R. Spriggs, Retrospective analysis of carboplatin and paclitaxel as initial second-line 
therapy for recurrent epithelial ovarian carcinoma: application toward a dynamic disease state model of ovarian cancer, J. Clin. Oncol. 20 (2002) 1238-1247; https://doi.org/ 10.1200/ JCO.2002.20.5.1238

10. M. U. Rashid, A. Zaidi, D. Torres, F. Sultan, A. Benner, B. Naqvi, A. R. Shakoori, A. Seidel-Renkert, H. Farooq, S. Narod, A. Amin and U. Hamann, Prevalence of BRCA1 and BRCA2 mutations in Pakistani breast and ovarian cancer patients, Int. J. Cancer 119 (2006) 2832-2839; https://doi. org/10.1002/ijc.22269

11. M. A. Moore, Y. Ariyaratne, F. Badar, Y. Bhurgri, K. Datta, A. Mathew, P. Gangadharan, A. Nandakumar, K. K. Pradhananga, M. H. Talukder, B. B. Yeole and T. Sobue, Cancer epidemiology in South Asia - past, present and future, Asian Pac. J. Cancer Prev. 11 (Suppl. 2) (2010) 49-66.

12. Y. Bhurgri, Karachi cancer registry data - implications for the National Cancer Control Program of Pakistan, Asian Pac. J. Cancer Prev. 5 (2004) 77-82.

13. Ovarian cancer second most common type in Pakistan, The Daily News 2016, https://www.thenews.com.pk/print/105779-Ovarian-cancer-second-most-common-type-in Pakistan; last access date July 15, 2018.

14. P. Cheema and R. Burkes, Overall survival should be the primary endpoint in clinical trials for advanced non-small-cell lung cancer, Curr. Oncol. 20 (2013) e150; https://doi.org/10.3747/co.20.1226

15. E. Eisenhauer, P. Therasse, J. Bogaerts, L. H. Schwartz, D. Sargent, R. Ford, J. Dancey, S. Arbuck, S. Gwyther, M. Mooney, L. Rubinstein, L. Shankar, L. Dodd, R. Kaplan, D. Lacombe and J. Verweij, New response evaluation criteria in solid tumours: revised RECIST guideline (version 1.1), Eur. J. Cancer 45 (2009) 228-247; https://doi.org/10.1016/j.ejca.2008.10.026

16. S. Zhang, F. Liang and I. Tannock, Use and misuse of common terminology criteria for adverse events in cancer clinical trials, BMC Cancer 16 (2016) Article ID 392 (6 pages); https://doi.org/10.1186/ s12885-016-2408-9

17. C. Aghajanian, S. V. Blank, B. A. Goff, P. L. Judson, M. G. Teneriello, A. Husain, M. A. Sovak, J. Yi and L. R. Nycum, OCEANS: a randomized, double-blind, placebo-controlled phase III trial of chemotherapy with or without bevacizumab in patients with platinum-sensitive recurrent epithelial ovarian, primary peritoneal, or fallopian tube cancer, J. Clin. Oncol. 30 (2012) 2039-2045; https://doi.org/10.1200/JCO.2012.42.0505

18. M. Kyrgiou, G. Salanti, N. Pavlidis, E. Paraskevaidis and J. P. Ioannidis, Survival benefits with diverse chemotherapy regimens for ovarian cancer: meta-analysis of multiple treatments, J. Natl. Cancer Inst. 98 (2006) 1655-1663; https://doi.org/10.1093/jnci/djj443

19. S. Pignata, G. Scambia, G. Ferrandina, A. Savarese, R. Sorio, E. Breda, V. Gebbia, P. Musso, L. Frigerio, P. Del Medico, A. V. Lombardi, A. Febbraro, P. Scollo, A. Ferro, S. Tamberi, A. Brandes, A. Ravaioli, M. R. Valerio, E. Aitini, D. Natale, L. Scaltriti, S. Greggi, C. Pisano, D. Lorusso, V. Salutari, F. Legge, M. Di Maio, A. Morabito, C. Gallo and F. Perrone, Carboplatin plus paclitaxel versus carboplatin plus pegylated liposomal doxorubicin as first-line treatment for patients with ovarian cancer: the MITO-2 randomized phase III trial, J. Clin. Oncol. 29 (2011) 3628-3635; https://doi.org/ 10.1200/JCO.2010.33.8566

20. R. S. Suidan, C. M. St Clair, S. J. Lee, J. N. Barlin, K. C. Long Roche, E. J. Tanner, Y. Sonoda, R. R. Barakat, O. Zivanovic and D. S. Chi, A comparison of primary intraperitoneal chemotherapy to consolidation intraperitoneal chemotherapy in optimally resected advanced ovarian cancer, Gynecol. Oncol. 134 (2014) 468-472; https://doi.org/10.1016/j.ygyno.2014.07.090

21. M. J. Piccart, K. Bertelsen, K. James, J. Cassidy, C. Mangioni, E. Simonsen, G. Stuart, S. Kaye, I. Vergote, R. B. R. Grimshaw, R. J. Atkinson, K. D. Swenerton, C. Trope, M. Nardi, J. Kaern, S. Tumolo, P. Timmers, J. Roy, F. Lhoas, B. Lindvall, M. Bacon, A. Birt, J. E. Andersen, B. Zee, J. Paul, B. Baron and S. Pecorelli, Randomized intergroup trial of cisplatin-paclitaxel versus cisplatin-cyclophosphamide in women with advanced epithelial ovarian cancer: three-year results, J. Natl. Cancer Inst. 92 (2000) 699-708; https://doi.org/10.1093/jnci/92.9.699 
22. A. du Bois, H. J. Lück, W. Meier, H. P. Adams, V. Möbus, S. Costa, T. Bauknecht, B. Richter, M. Warm, W. Schröder, S. Olbricht, U. Nitz, C. Jackisch, G. Emons, U. Wagner, W. Kuhn and J. Pfisterer, A randomized clinical trial of cisplatin/paclitaxel versus carboplatin/paclitaxel as first-line treatment of ovarian cancer, J. Natl. Cancer Inst. 95 (2003) 1320-1329; doi 10.1093/jnci/djg036

23. R. F. Ozols, B. N. Bundy, B. E. Greer, J. M. Fowler, D. Clarke-Pearson, R. A. Burger, R. S. Mannel, K. DeGeest, E. M. Hartenbach and R. Baergen, Phase III trial of carboplatin and paclitaxel compared with cisplatin and paclitaxel in patients with optimally resected stage III ovarian cancer: a Gynecologic Oncology Group study, J. Clin. Oncol. 21 (2003) 3194-3200; https://doi.org/10.1200/ jco.2003.02.153

24. M. Fung-Kee-Fung, T. Oliver, L. Elit, A. Oza, H. W. Hirte and P. Bryson, Optimal chemotherapy treatment for women with recurrent ovarian cancer, Curr. Oncol. 14 (2007) 195-208.

25. Y. Wang, J. Herrstedt, H. Havsteen, R. DePoint Christensen, M. R. Mirza, B. Lund, J. Maenpaa and G. Kristensen, A multicenter, non-randomized, phase II study of docetaxel and carboplatin administered every 3 weeks as second line chemotherapy in patients with first relapse of platinum sensitive epithelial ovarian, peritoneal or fallopian tube cancer, BMC Cancer 14 (2014) Article ID 937 (7 pages); https://doi.org/10.1186/1471-2407-14-937

26. L. A. Holmberg, P. Paley and B. Goff, Docetaxel and carboplatin as salvage therapy for first-relapsed, platinum-sensitive, stage III/IV advanced ovarian cancer, Clin. Ovar. Other Gynecol. Cancer 7 (2014) 13-17; https://doi.org/10.1016/j.cogc.2014.12.005

27. J. M. Gibson, S. Alzghari, C. Ahn, H. Trantham and N. M. La-Beck, The role of pegylated liposomal doxorubicin in ovarian cancer: a meta-analysis of randomized clinical trials, Oncologist 18 (2013) 1022-1031; https://doi.org/ 10.1634/theoncologist.2013-0126

28. M. Parmar, J. Ledermann, N. Colombo, A. du Bois, J. F. Delaloye, G. B. Kristensen, S. Wheeler, A. M. Swart, W. Qian, V. Torri, I. Floriani, G. Jayson and A. Lamont, C. Tropé, Paclitaxel plus platinum-based chemotherapy versus conventional platinum-based chemotherapy in women with relapsed ovarian cancer: the ICON4/AGO-OVAR-2.2 trial, Lancet 361 (2003) 2099-2106; https://doi. org/10.1016/S0140-6736(03)13718-X

29. J. Pfisterer, M. Plante, I. Vergote, A. du Bois, H. Hirte, A. J. Lacave, U. Wagner, A. Stähle, G. Stuart, R. Kimmig, S. Olbricht, T. Le, J. Emerich, W. Kuhn, J. Bentley, C. Jackisch, H. J. Lück, J. Rochon, A. H. Zimmermann and E. Eisenhauer, Gemcitabine plus carboplatin compared with carboplatin in patients with platinum-sensitive recurrent ovarian cancer: an intergroup trial of the AGO-OVAR, the NCIC CTG, and the EORTC GCG, J. Clin. Oncol. 24 (2006) 4699-4707; https://doi. org/10.1200/JCO.2006.06.0913

30. D. Bafaloukos, H. Linardou, G. Aravantinos, C. Papadimitriou, A. Bamias, G. Fountzilas, H. P. Kalofonos, P. Kosmidis, E. Timotheadou, T. Makatsoris, E. Samantas, E. Briasoulis, C. Christodoulou, P. Papakostas, D. Pectasides and A. M. Dimopoulos, A randomized phase II study of carboplatin plus pegylated liposomal doxorubicin versus carboplatin plus paclitaxel in platinum sensitive ovarian cancer patients: a Hellenic Cooperative Oncology Group study, BMC Med. 8 (2010) Article ID 3 (12 pages); https://doi.org/10.1186/1741-7015-8-3

31. C. J. Dunton, Management of treatment-related toxicity in advanced ovarian cancer, Oncologist 7 (Suppl. 5) (2002) 11-19.

32. K. Kudoh, M. Takano, H. Kouta, R. Kikuchi, T. Kita, M. Miyamoto, A. Watanabe, M. Kato, T. Goto and Y. Kikuchi, Effects of bevacizumab and pegylated liposomal doxorubicin for the patients with recurrent or refractory ovarian cancers, Gynecol. Oncol. 122 (2011) 233-237; https://doi. org/10.1016/j.ygyno.2011.04.046 


\section{Supplementary Material}

Table SI. Chemotherapy regimens used in Shaukat Khanum Memorial Cancer and Research Hospital for patients with ovarian cancer

Chemotherapy regimen Dose and cycles

Number of

patients

(total, $n=251$ )

Paclitaxel (PTX) Weekly paclitaxel $80 \mathrm{mg} \mathrm{m}^{-2} \mathrm{IV}$ over 1 hour

Topotecan (TOPO)

Topotecan $1.25-1.5 \mathrm{mg} \mathrm{m}^{-2}$ as single agent IV over 30 minutes on days 1-5; every 21 days

Carboplatin + liposomal Carboplatin AUC 5 IV push plus liposomal doxorubicin doxorubicin (CLD) $\quad 30 \mathrm{mg} \mathrm{m}^{-2}$ IV over 30 minutes; every 28 days for six cycles

Gemcitabine $1000 \mathrm{mg} \mathrm{m}^{-2}$ IV over 30 minutes on day 1

Carboplatin + gemcitabine and 8 plus carboplatin AUC 4-5 IV over 1 hour on day 1; every 21 days for six cycles

Carboplatin + paclitaxel

Paclitaxel $175 \mathrm{mg} \mathrm{m}^{-2}$ i.v. over 3 hours plus carboplatin

(AUC 5-7.5) over 30 minutes on day 1; every 21 days for six cycles

Docetaxel $75 \mathrm{mg} \mathrm{m}^{-2} \mathrm{IV}$ over 1 hour plus carboplatin

Carboplatin + docetaxel

(AUC 5) IV over 1 hour on day 1; every 21 days up to six cycles

Gemcitabine $1000 \mathrm{mg} \mathrm{m}^{-2}$ IV over 30 minutes on day 1 and

Carboplatin + gemcitabine

8 plus carboplatin AUC 4-5 IV push on day 1; every 21

+ bevacizumab (CGB)

days for 6 cycles plus bevacizumab $15 \mathrm{mg} \mathrm{kg}^{-1}$ IV on day 1 prior to gemcitabine and carboplatin and continued until disease progression or unbearable toxicity

Table SII. Response evaluation criteria in solid tumors (RECIST)

Complete response (CR)

Disappearance of all target lesions. Any pathological lymph nodes (whether target or non-target) must have reduction in short axis to $<10 \mathrm{~mm}$.

Partial response (PR)

At least a $30 \%$ decrease in the sum of diameters of target lesions, taking as reference the baseline sum diameters.

At least a $20 \%$ increase in the sum of diameters of target lesions, taking

Progressive disease (PD) as reference the smallest sum on study. In addition to the relative increase of $20 \%$, the sum must also demonstrate an absolute increase of at least 5 $\mathrm{mm}$. The appearance of one or more lesions is also considered progression. 


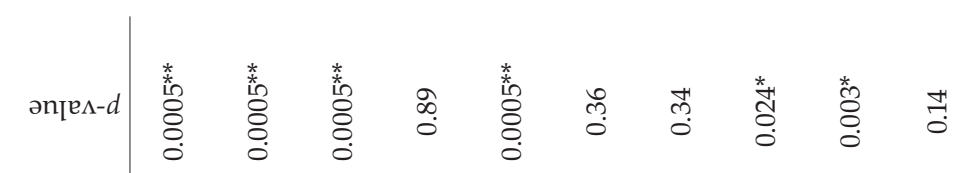

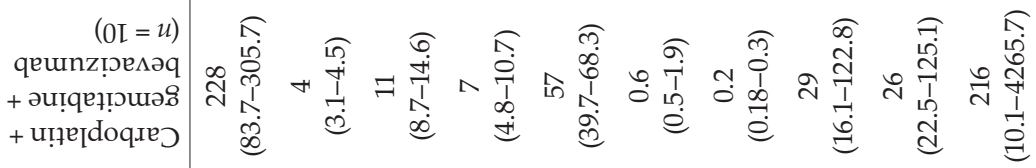


S. Sattar et al:: Comparison of clinico-pathological characteristics and survival of recurrent ovarian cancer patients on seven different chemo-protocols, Supplementary Material, Acta Pharm. 69 (2019) S1-S4.

Table SIV. Toxicity grading (as per NCI-CTC) according to chemotherapy regimens

\begin{tabular}{|c|c|c|c|c|c|c|c|}
\hline Toxicity & 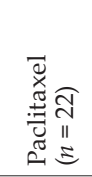 & 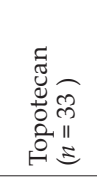 & 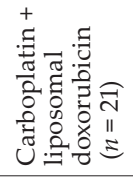 & 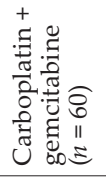 & 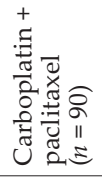 & 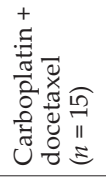 & 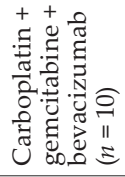 \\
\hline \multicolumn{8}{|c|}{ Toxicity grading $(n, \%)$} \\
\hline \multicolumn{8}{|c|}{ Hematological } \\
\hline \multicolumn{8}{|c|}{ Anemia } \\
\hline Grade 1 & $10(46)$ & $6(18)$ & $7(33)$ & $18(30)$ & $45(50)$ & $3(20)$ & $7(70)$ \\
\hline Grade 2 & $1(4)$ & $13(39)$ & $7(33)$ & $22(37)$ & $24(27)$ & $7(47)$ & $2(20)$ \\
\hline Grade 3 & - & $2(6)$ & - & $9(15)$ & - & $1(7)$ & - \\
\hline \multicolumn{8}{|c|}{ Leukopenia } \\
\hline Grade 1 & $4(18)$ & $1(3)$ & $2(10)$ & $9(15)$ & $14(16)$ & $1(7)$ & $1(10)$ \\
\hline Grade 2 & $1(4)$ & $1(3)$ & $2(10)$ & $8(13)$ & $2(2)$ & - & $1(10)$ \\
\hline Grade 3 & 0 & - & - & $1(2)$ & $1(1)$ & - & - \\
\hline Grade 4 & $1(4)$ & - & - & - & - & - & - \\
\hline \multicolumn{8}{|c|}{ Thrombocytopenia } \\
\hline Grade 1 & - & $1(3)$ & $4(19)$ & $11(18)$ & $11(12)$ & $7(47)$ & $3(30)$ \\
\hline Grade 2 & - & - & - & $2(3)$ & $2(2)$ & - & - \\
\hline Grade 3 & - & - & - & $1(2)$ & $1(1)$ & - & $1(10)$ \\
\hline Grade 4 & - & - & - & $1(2)$ & - & - & - \\
\hline \multicolumn{8}{|c|}{ Neutropenia } \\
\hline Grade 1 & $4(18)$ & - & $3(14)$ & $10(17)$ & $10(11)$ & $1(7)$ & $1(10)$ \\
\hline Grade 2 & $2(9)$ & $4(12)$ & $3(14)$ & $13(22)$ & $7(8)$ & - & $1(10)$ \\
\hline Grade 3 & - & $1(3)$ & $1(5)$ & $4(6)$ & $1(1)$ & - & $2(20)$ \\
\hline Grade 4 & - & - & - & - & $1(1)$ & - & - \\
\hline \multicolumn{8}{|c|}{ Renal toxicity } \\
\hline \multicolumn{8}{|c|}{ Creatinine } \\
\hline Grade 1 & - & $6(18)$ & - & $3(5)$ & $3(3)$ & $1(7)$ & - \\
\hline Grade 2 & - & - & - & $1(2)$ & $2(2)$ & - & - \\
\hline \multicolumn{8}{|c|}{ Hepatic toxicity } \\
\hline \multicolumn{8}{|c|}{ Bilirubin } \\
\hline Grade 1 & - & - & $1(5)$ & - & - & - & - \\
\hline Grade 2 & - & - & $1(5)$ & - & $1(1)$ & - & - \\
\hline Grade 4 & - & - & - & $1(2)$ & - & - & - \\
\hline \multicolumn{8}{|c|}{ GIT events } \\
\hline \multicolumn{8}{|c|}{ Abdominal pain } \\
\hline Grade 1 & $1(5)$ & $2(6)$ & $1(5)$ & 10 (17) & $5(6)$ & $3(20)$ & $4(40)$ \\
\hline Grade 2 & - & - & - & $3(5)$ & - & - & - \\
\hline \multicolumn{8}{|c|}{ Abdominal distension } \\
\hline Grade 1 & $2(9)$ & - & $2(10)$ & $5(8)$ & $2(2)$ & $5(33)$ & - \\
\hline \multicolumn{8}{|c|}{ Constipation } \\
\hline Grade 1 & - & $4(12)$ & - & $4(7)$ & $3(3)$ & - & - \\
\hline \multicolumn{8}{|l|}{ Nausea } \\
\hline Grade 1 & $1(5)$ & $1(3)$ & $1(5)$ & $4(7)$ & $9(10)$ & $2(13)$ & $4(40)$ \\
\hline
\end{tabular}


S. Sattar et al:: Comparison of clinico-pathological characteristics and survival of recurrent ovarian cancer patients on seven different chemo-protocols, Supplementary Material, Acta Pharm. 69 (2019) S1-S4.

\begin{tabular}{|c|c|c|c|c|c|c|c|}
\hline Grade 2 & - & - & - & - & - & - & - \\
\hline Grade 3 & - & $3(9)$ & - & $1(2)$ & $1(1)$ & - & - \\
\hline \multicolumn{8}{|l|}{ Vomiting } \\
\hline Grade 1 & - & $2(6)$ & $1(5)$ & $9(15)$ & $3(3)$ & - & - \\
\hline Grade 2 & - & - & $1(5)$ & $4(7)$ & - & - & - \\
\hline \multicolumn{8}{|l|}{ Diarrhea } \\
\hline Grade 1 & - & $1(3)$ & - & $5(8)$ & $2(2)$ & $1(7)$ & - \\
\hline Grade 2 & - & $2(6)$ & - & $2(3)$ & $1(1)$ & - & - \\
\hline Grade 3 & - & - & - & $1(2)$ & $1(1)$ & - & - \\
\hline \multicolumn{8}{|l|}{ Anorexia } \\
\hline Grade 1 & - & $1(3)$ & $1(5)$ & $2(3)$ & $4(4)$ & $1(7)$ & - \\
\hline Grade 2 & - & $1(3)$ & $1(5)$ & $1(2)$ & - & - & - \\
\hline \multicolumn{8}{|c|}{ Peripheral neuropathy } \\
\hline Grade 1 & $4(18)$ & $1(3)$ & $3(14)$ & $4(7)$ & $16(18)$ & $1(7)$ & - \\
\hline Grade 2 & $2(9)$ & $1(3)$ & $1(5)$ & - & $4(4)$ & - & - \\
\hline Grade 3 & - & $2(6)$ & - & - & $2(2)$ & - & - \\
\hline \multicolumn{8}{|l|}{ Fatigue } \\
\hline Grade 1 & $3(14)$ & $3(9)$ & $6(29)$ & $12(20)$ & $10(11)$ & $6(7)$ & $4(40)$ \\
\hline Grade 2 & - & $1(3)$ & - & $2(3)$ & $2(2)$ & - & - \\
\hline Grade 3 & - & - & $1(5)$ & - & - & - & - \\
\hline \multicolumn{8}{|c|}{ Pulmonary toxicity } \\
\hline \multicolumn{8}{|c|}{ Cough } \\
\hline Grade 1 & - & $2(6)$ & $2(2)$ & $5(8)$ & $6(7)$ & - & $4(40)$ \\
\hline Grade 2 & - & - & $3(14)$ & - & - & - & - \\
\hline \multicolumn{8}{|c|}{ Cardiovascular events } \\
\hline \multicolumn{8}{|c|}{ Hypertension } \\
\hline Grade 1 & - & - & - & $1(2)$ & $2(2)$ & - & $1(10)$ \\
\hline Grade 2 & - & - & - & - & - & - & $3(30)$ \\
\hline \multicolumn{8}{|c|}{ Tachycardia } \\
\hline Grade 1 & - & $1(3)$ & - & $3(5)$ & $1(1)$ & - & - \\
\hline \multicolumn{8}{|l|}{ Others } \\
\hline \multicolumn{8}{|c|}{ Mucositis } \\
\hline Grade 1 & - & - & - & $3(5)$ & $3(3)$ & $1(2)$ & $3(30)$ \\
\hline Grade 2 & - & - & - & - & $1(1)$ & - & - \\
\hline \multicolumn{8}{|l|}{ Rash } \\
\hline Grade 1 & - & - & - & $4(7)$ & $1(1)$ & $2(13)$ & $1(10)$ \\
\hline \multicolumn{8}{|c|}{ Depression } \\
\hline Grade 1 & $2(9)$ & - & - & - & $2(2)$ & - & - \\
\hline Grade 2 & - & - & $2(2)$ & - & - & - & - \\
\hline \multicolumn{8}{|l|}{ Fever } \\
\hline Grade 1 & - & $2(6)$ & - & - & $1(1)$ & - & $2(20)$ \\
\hline \multicolumn{8}{|l|}{ Bleeding } \\
\hline Grade 1 & - & $2(6)$ & - & - & - & - & - \\
\hline \multicolumn{8}{|c|}{ Infection during therapy } \\
\hline Yes & - & $1(3)$ & $6(29)$ & $3(5)$ & $18(20)$ & $1(7)$ & $1(10)$ \\
\hline No & - & - & - & - & - & - & - \\
\hline
\end{tabular}

NCI-CTC - National Cancer Institute Common Toxicity Criteria 